\title{
Cambio de pareja progenitora en puérperas de un hospital de la Región Metropolitana. Frecuencia y factores asociados
}

\author{
Nelson A Vargas C, Cristián Villar B, Alejandro Lastra P. \\ Change in reproductive partner \\ among women delivering at a \\ Metropolitan Santiago public \\ hospital
}

Background: Previus studies in Metropolitan Santiago, showed that at least $10 \%$ of mothers changed their reproductive partner. Aim. To study the frequency of reproductive partner change and its associated factors among women delivering in a public hospital. Subjects and methods: We interviewed 859 non primiparous women that gave birth to a child in a public hospital of Metropolitan Santiago, between March 2001 and February 2002. Results: Mean maternal age rose according to birth order from 27.4 to 33.6 years from the second to the fourth sibling. Married women were the largest group and single ones decreased from the second to the third sibling. Twenty two percent had some activity out of home and those with a high birth order (fourth or more) exhibited poorer schooling. Unwanted pregnancies increased with birth order, reaching 55\% among fourth and over» births. Thirty four percent of women delivering their second child, changed their partner. Among women delivering their third child, $38.6 \%$ had one sibling with a different father; $20.4 \%$ had changed partner during their second pregnancy and 20\% during the current one. Among those in the fourth and over» group, $56.1 \%$ had at least one sibling with a different father; $18.3 \%$ had changed partner in the second sibling; $15 \%$, in the third and $27.8 \%$, in the fourth and over. In all birth orders, mothers with activity out of home exhibited a higher frequency of partner change. The most common reasons to change partner were infidelity, abandon or a personal decision, while economic reasons were uncommon. Conclusions: A great number of children are exposed to bonding ruptures and to the consequential mental health or behavioral problems (Rev Méd Chile 2004; 132: 719-24).

(Key Words: Parenthood status; Sexual partners; Single parent)

Recibido el 30 de septiembre, 2003. Aceptado en versión corregida el 5 de abril, 2004. Departamento de Pediatría y Cirugía Infantil Occidente, Facultad de Medicina, Universidad de Chile.

Correspondencia a: Nelson A Vargas. Casilla 33052. Correo

33. Santiago de Chile. Teléfono fax: (562) 681-5217.

E-mail: nvargas@med.uchile.cl 
L a a vida en pareja ha sido, desde tiempos remotos, la base de la familia y, por ende, de la sociedad. La pareja es una unidad social básica cuya estabilidad repercutiría positivamente en la salud psíquica, social y aun biológica de la familia; mientras sus rupturas y cambios impactarían negativamente ${ }^{1}$.

Durante el siglo XX, el mundo y nuestro país experimentaron una transición demográfica con una fase inicial de gran descenso de la mortalidad y la natalidad; seguida rápidamente por otra en que ocurren cambios relevantes en la familia ${ }^{2}$. Al comienzo del siglo XXI, el descenso de la natalidad parece mantenerse, unido a un importante incremento de niños nacidos fuera del matrimonio $^{3}$. Adicionalmente, estudios hechos en 1993 en puérperas del área occidente de Santiago mostraron que el cambio de pareja progenitora ocurría, al menos, en $10 \%$ de ellas ${ }^{4}$.

Estos antecedentes proporcionaron el propósito de este estudio: recoger información sobre el cambio de pareja; precisando su frecuencia en puérperas no primíparas de un hospital de la Región Metropolitana y midiendo factores asociados. Los objetivos fueron caracterizar a las mujeres según edad, estado civil, escolaridad y número de orden del embarazo y establecer la proporción que declaraba que el embarazo no había sido deseado.

Se midió la frecuencia del cambio y sus variaciones según número de orden del embarazo y actividad materna dentro y fuera del hogar, precisando los motivos esgrimidos para explicarlo.

\section{PERSONAS Y MÉTODOS}

Se encuestaron, de preferencia en el momento del alta médica, 859 puérperas no primíparas de la Maternidad del Hospital San Juan de Dios (Occidente de Santiago), entre marzo de 2001 y febrero de 2002. Se entrevistaron mujeres con hijos vivos, excluyendo las que se negaron a participar o que tuviesen dolor o alguna condición médica que impidiese responder. Sólo hubo nueve exclusiones.

El número de entrevistas necesarias para estudiar el cambio de pareja según orden de nacimiento se definió así: con el anuario «emografía 1998», del Instituto Nacional de Estadística² ${ }^{2}$ se procedió a calcular la frecuencia estimada de los distintos órdenes de nacimiento en el país, a contar del segundo y hasta la clase «cuarto y más». Enseguida, se calculó el número esperado de nacimientos en la maternidad durante 2001, utilizando la cifra de partos de 1999 ( $\mathrm{n}=5.164)$; sabiendo que por el descenso de la natalidad del país era razonable esperar una reducción en el número. A esta cifra esperada se le aplicó la distribución porcentual según orden de nacimiento observada en Chile. A continuación, utilizando el Programa StatCalc, de Epiinfo 5,1, se calculó la muestra estratificada por orden de nacimiento, esperando $8 \%$ de frecuencia de cambio de pareja en cualquier orden de nacimiento, un peor resultado esperable de $5 \%$ y un intervalo de confianza de 95\%. La frecuencia esperada de cambio se obtuvo de un estudio previo ${ }^{3}$, de 1993, en el Hospital Félix Bulnes Cerda, de la misma área de salud. El procedimiento calculó que las encuestas necesarias para detectar el cambio de pareja en las condiciones fijadas eran: 271, hijos segundos; 230, terceros y 195, cuarto o más; conformando un total de 696.

Se consideró cambio de pareja el tener un hijo de padre diferente al de los hermanos y, para estudiarlo detalladamente, se preguntó éste para cada embarazo de la vida reproductiva.

Para estudiar el impacto de trabajar fuera del hogar, se separó a las mujeres en dos grupos, según si salían o no del mismo. En la primera categoría se incluyó a las estudiantes y trabajadoras manuales y técnicas.

El análisis estadístico de las diferencias porcentuales se hizo mediante el cálculo de $\mathrm{Z}$, con un nivel de significación de alfa $=0,05$, sin usar paquetes estadísticos.

\section{Resultados}

Se encuestó 859 madres: 399, secundíparas (46,4\%); 280, de tercer orden de paridad (32,6\%) y 180 (21\%) de orden cuarto y más. Estas cifras superan las estimadas como necesarias; salvo en el último grupo, difícil de ubicar por su menor frecuencia.

La Tabla 1 muestra la edad y estado civil maternos de los grupos estudiados. La edad se elevó 6,2 años entre el hijo segundo y el «cuarto o más». En el estado civil, predominaron las que 
dijeron ser casadas; la proporción de solteras descendió significativamente entre el segundo y tercer hijo ( $\mathrm{p}:<0,05)$ y las convivientes tuvieron frecuencia relativamente similar en los tres grupos.

La Tabla 2 presenta la escolaridad materna. Se pesquisó a nueve analfabetas (1\%) y las mujeres de orden de nacimiento «cuarto y más» tuvieron peor escolaridad. En ellas, el nivel inferior a medio llegó a cifras significativamente superiores $(p:<0,05)$ a las observadas en los otros órdenes de paridad.

Frente a la pregunta: «Usted, quería quedar embarazada de este niño?» sólo 423 (49,2\%) dijeron que sí; otras $253(29,5 \%)$ dijeron que no y 183 $(21,3 \%)$ dijeron no estar seguras o no saberlo bien.

Tabla 1. Edad y estado civil de las puérperas entrevistadas según número de orden del niño

\begin{tabular}{|c|c|c|c|c|c|}
\hline & \multicolumn{5}{|c|}{ Número de orden del niño } \\
\hline Edad (años) & $\mathrm{n}=399$ & 280 & & 18 & \\
\hline Promedio & 27,4 & 31 & & & 3 \\
\hline Desviación estándar & 5,4 & & & & , 2 \\
\hline Mediana & 27,0 & 31 & & & 1,0 \\
\hline Mínima & 17,0 & 18 & & & 0 \\
\hline Máxima & 43,0 & 45 & & &, 0 \\
\hline \% con 40 y más años & 2,3 & & & & 3 \\
\hline Estado civil & $\mathrm{n} \quad \%$ & $\mathrm{n}$ & $\%$ & $\mathrm{n}$ & $\%$ \\
\hline Casada & 51,8 & 157 & 57,1 & 87 & 50,0 \\
\hline Soltera & $19,2 *$ & 33 & 12,0 & 20 & 11,5 \\
\hline Conviviente & 29,0 & 82 & 29,8 & 57 & 32,8 \\
\hline Viuda & 0 & 0 & - & 2 & 1,1 \\
\hline Separada & 0 & 3 & 1,1 & 8 & 4,5 \\
\hline No responde & $(3,3)$ & 5 & $(2,0)$ & (6) & $(3,3)$ \\
\hline
\end{tabular}

${ }^{*}$ p: $<0,05$. La proporción de solteras es mayor en este grupo.

Tabla 2. Escolaridad según número de orden del niño

\begin{tabular}{|c|c|c|c|c|c|c|}
\hline \multirow[t]{2}{*}{ Escolaridad } & \multicolumn{5}{|c|}{ Número de orden del niño } & Cuarto y más \\
\hline & $\mathrm{n}$ & $\%$ & $\mathrm{n}$ & $\%$ & $\mathrm{n}$ & $\%$ \\
\hline Analfabetas & 4 & 1,0 & 2 & 0,7 & 3 & 1,7 \\
\hline Lee y escribe & 0 & - & 0 & - & 5 & 2,8 \\
\hline Básica incompleta & 42 & 10,6 & 42 & 15,0 & 54 & 30,0 \\
\hline Básica completa & 68 & 17,1 & 43 & 15,4 & 24 & 13,3 \\
\hline Subtotal & 114 & 28,7 & 87 & 31,1 & 86 & $47,7^{*}$ \\
\hline Media incompleta & 106 & 26,7 & 83 & 29,6 & 52 & 28,9 \\
\hline Media completa & 129 & 32,5 & 73 & 26,1 & 30 & 16,7 \\
\hline Técnica incompleta & 2 & 0,5 & 2 & 0,7 & 4 & 2,2 \\
\hline Técnica completa & 33 & 8,3 & 20 & 7,1 & 4 & 2,2 \\
\hline Universitaria o superior & 13 & 3,3 & 15 & 5,4 & 4 & 2,2 \\
\hline Ignorada & (2) & $(1,0)$ & 0 & - & 0 & - \\
\hline
\end{tabular}

$*_{p}$ : $<0,05$. Hay más mujeres con educación bajo el nivel medio. 
La cifra de quienes dijeron no haberlo deseado subió al aumentar el número de orden: $28,7 \%$ en las secundíparas; $49,8 \%$, en las de tercer parto y $55,3 \%$, en el orden más alto. La diferencia fue significativa (Z: 5,57; $\mathrm{p} \varangle 0,0002$ ) al comparar secundíparas y las de orden tercero; pero no lo fue al comparar este último grupo con el orden «cuarto y más».

Cambio de pareja. El 40,4\% $(n=344)$ de las 859 mujeres dijeron tener al menos un hijo de padre distinto al de sus hermanos y en el embarazo actual, 27,4\% había cambiado de pareja (235/859).

Cambio en el segundo hijo. Para el embarazo actual las 399 secundíparas habían cambiado de pareja en $33,8 \%(n=135)$.

El total de 859 mujeres (incluyendo las no secundíparas) había tenido $26,2 \%$ de cambio en su segunda gestación (225/859).

Cambio en el tercer hijo. En las 280 puérperas de tercer hijo, 38,6\% (n=108) tenía algún hijo de padre distinto; 20,4\% (57/280) había cambiado pareja en el segundo niño y $20 \%$ (56/280), en el tercero. La suma de estos porcentajes es superior a $38,6 \%$ porque algunas habían reconstruido su relación con el padre del primer hijo. Durante su vida reproductiva estas mujeres habían tenido 840 partos y 113 cambios de pareja (13,5\%, de los partos), algunos para reconstituir relaciones (113 cambios con 108 padres).
En el total de 859 encuestadas, 460 mujeres había tenido terceras gestaciones, en las que el cambio llegó a 18\% (83/460).

Cambio en el hijo cuarto y más. En 180 puérperas, $56,1 \%(\mathrm{n}=101)$ tenía algún hijo de padre distinto; $18,3 \%(n=33)$ había cambiado en el segundo niño; $15 \%(n=27)$, en el tercero y $27,8 \%$ $(n=50)$, en el cuarto o más.

Los hijos segundos y de orden cuarto y más tenían la más alta frecuencia de cambio. Algunas madres habían reconstruido su relación con parejas anteriores, lo que explica que la suma de los porcentajes de cambio sea de $61,1 \%$; mientras los padres distintos sólo alcanzan al 56,1\%.

Cambios según la permanencia en casa. En lo referente a actividad, sólo cuatro madres de segunda paridad omitieron la respuesta. El 21,8\% declaró alguna actividad fuera del hogar $\mathrm{y}$, en todos los órdenes de nacimiento, la edad fue similar entre quienes salían o permanecían en casa.

Salían de casa para actividades laborales y de estudio 23,8\% (94/395) de las secundíparas; $21 \%$ (59/280) en la tercera paridad, y 18,9\% (34/180) en las de orden mayor. La edad y frecuencia del cambio de pareja en estos subgrupos se presenta en la Tabla 3. En todos los órdenes de paridad el grupo con actividad fuera de casa tuvo mayor frecuencia de cambio, con diferencias significativas en los tres grupos de puérperas ( $\mathrm{p}$ : $<0,05)$.

Tabla 3. Frecuencia del cambio de pareja y edad según número de orden, entre quienes tienen actividades dentro y fuera de la casa

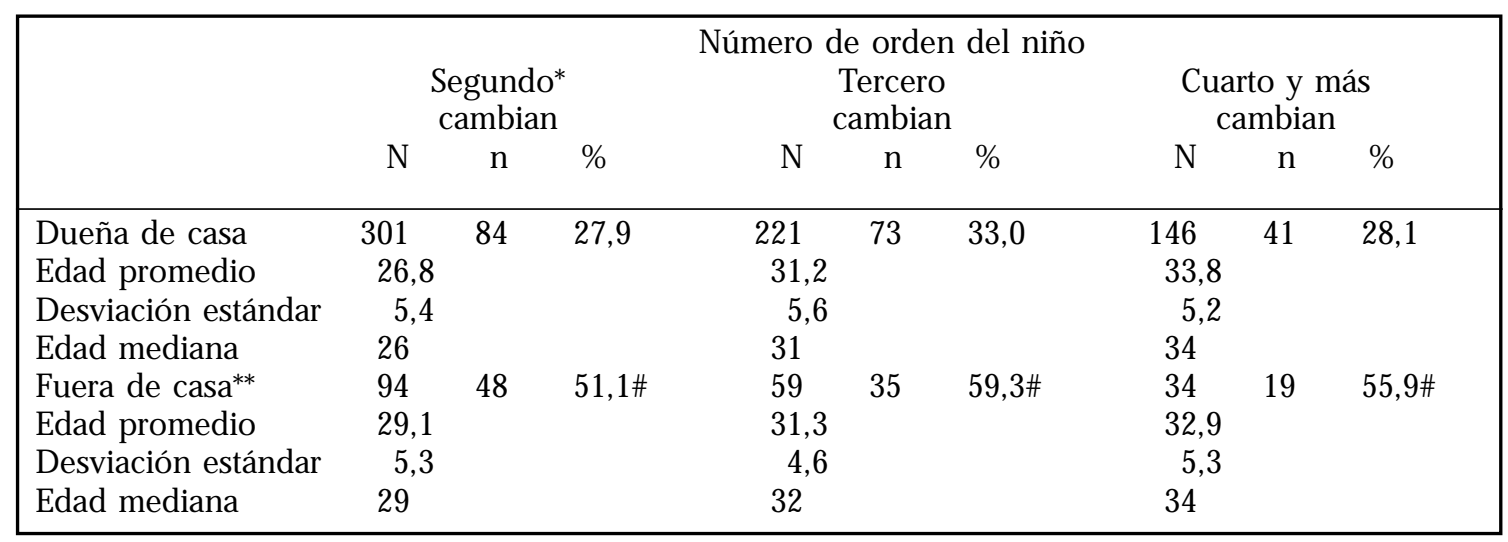

*Cuatro encuestadas no contestaron su tipo de actividad. **Incluye estudiantes y mujeres con trabajo, aunque sean dueñas de casa.

$\# p$ : $<0,05$. En todos los órdenes de paridad hubo mayor frecuencia de cambio en quienes tenían actividad fuera de casa. 
Razones para el cambio. Esta pregunta fue respondida por casi la totalidad de las entrevistadas y muchas mujeres dieron más de una causa (Tabla 4). La infidelidad o abandono de la pareja o de la misma entrevistada fue la causa más invocada, pero con tendencia a descender al aumentar el número de orden. Así, en el grupo de orden mayor sólo superó ligeramente a la segunda causa más frecuente: la violencia. Ésta, en cambio, mostró tendencia al aumento al subir el número de orden; mientras la inestabilidad de la pareja -tercera en frecuenciadescendió en frecuencia después del segundo hijo.

El consumo de tóxicos lícitos e ilícitos, en cambio, tuvo un leve ascenso desde el segundo al tercer hijo, para mantenerse más bien estable, entre el tercero y cuarto y más.

Las razones económicas fueron relativamente infrecuentes y oscilaron sin una tendencia clara; mientras que la muerte de la pareja se incrementó al subir el número de orden.

\section{DisCUSIÓN}

Limitaciones del estudio. Los datos se obtuvieron por declaración de las madres, careciendo de confirmación. Eventualmente, esto puede sesgar algunas respuestas; en especial, la razón del cambio, obtenida en forma unilateral, sin la perspectiva del padre. Otras respuestas que pueden verse afectadas por la falta de confirmación son la escolaridad y el estado civil.
Una segunda limitación es no haber alcanzado el número de encuestas estimado como necesario en las puérperas de orden cuarto y más. El déficit de 15 encuestas se pudiese atribuir a la disminución de la natalidad chilena, concretado especialmente en mujeres de orden alto de nacimiento. El cálculo de encuestas necesarias se hizo con datos de 1998 (únicos oficiales disponibles en ese momento); mientras la aplicación ocurrió tres a cuatro años después. De esta manera, no creemos que el déficit de $7,7 \%$ en el número deseado para ese grupo afecte de manera fundamental la información de éste.

Una impresión general, que deja la caracterización de las mujeres estudiadas, es que ellas representarían en medida apreciable a la población urbana metropolitana femenina en proceso reproductivo y que se atiende en el sector público. No obstante, llama la atención el 1\% de analfabetismo, en la Región Metropolitana de un país que ha tenido grandes mejorías en su nivel educacional.

En el lapso de 100 años, Chile ha experimentado un gran progreso en la mortalidad infantil. Parte importante de éste pudiese atribuirse a mejores condiciones y resultados del proceso reproductivo, que se concentró en edades maternas de menor riesgo; mientras los embarazos ocurrían después de espaciamientos gradualmente más largos. Este estudio constató algunos hechos considerados beneficiosos, propios de estos cambios demográficos: no hubo diferencia menor de dos años y mayor de cinco en los promedios 0

Tabla 4. M otivos del cambio de pareja según número de orden

\begin{tabular}{|c|c|c|c|c|c|c|}
\hline \multirow[b]{4}{*}{ Madres que cambian } & \multicolumn{6}{|c|}{ Número de orden del niño } \\
\hline & \multicolumn{2}{|c|}{ Segundo } & \multicolumn{2}{|c|}{ Tercero } & \multicolumn{2}{|c|}{ Cuarto y más } \\
\hline & $\mathrm{n}$ & $\%$ & $\mathrm{n}$ & $\%$ & $\mathrm{n}$ & $\%$ \\
\hline & 225 & 100,0 & 83 & 100,0 & 48 & 100,0 \\
\hline No responde & 2 & 0,9 & 0 & 0,0 & 1 & 2,1 \\
\hline Infidelidad conyugal & 123 & 54,7 & 35 & 42,2 & 17 & 35,4 \\
\hline Violencia & 51 & 22,7 & 22 & 26,5 & 15 & 31,2 \\
\hline La pareja era ocasional y no estable & 33 & 14,7 & 6 & 7,2 & 2 & 4,2 \\
\hline Alcohol & 28 & 12,4 & 15 & 18,1 & 8 & 16,7 \\
\hline Drogas & 19 & 8,4 & 5 & 6,0 & 4 & 8,3 \\
\hline Razones económicas & 10 & 4,4 & 6 & 7,2 & 3 & 6,2 \\
\hline Muerte de la pareja & 8 & 3,6 & 4 & 4,8 & 5 & 10,4 \\
\hline Otras & 29 & 12,9 & 10 & 12,0 & 2 & 4,2 \\
\hline
\end{tabular}


medianas de edad materna entre los distintos órdenes de nacimiento. Estos datos permiten plantear que el espaciamiento intergenésico puede estar favoreciendo, en términos generales, un buen proceso reproductivo, al no ser excesivamente corto ni largo.

Hallazgo negativo es la cifra de embarazos no deseados que, aun en el grupo con la frecuencia menor, llegó a 28,6\%. Esta cifra, en mujeres de educación relativamente favorable y de un medio donde se supone que hay acceso al control de la natalidad, indica que la reproducción no se realiza de acuerdo al deseo de las entrevistadas.

La segunda fase de la transición demográfica ha estado marcada por los cambios en la familia, destacando el nacimiento fuera del matrimonio y la inestabilidad familiar. La segunda, aquí explorada, sorprende por su magnitud. Esta inestabilidad repercutiría en la puericultura, la salud mental de los niños y, eventualmente, en la salud biológica. Estas repercusiones posibles obligan a estudiar el cambio de pareja que, en este estudio, llegó a frecuencias sorprendentes: ya en las madres que terminaban el segundo embarazo un tercio tenía un hijo con hermano de padre distinto y en el grupo de orden mayor el fenómeno llegó al $56,1 \%$. Naturalmente, ese grupo ha tenido más embarazos y, por ello, más ocasiones para cambiar su pareja.

\section{REFERENCIAS}

1. Donoso C. Familia y Salud Mental. En: Imágenes en Salud Mental. 28-32. Ed. Laser. Santiago de Chile. 1999.

2. VARGAS NA. Cambios en la familia: repercusiones en la práctica pediátrica. Rev Chil Pediatr 2001; 72: 77-80.

3. Demografía 1998. Instituto Nacional de Estadística. 2000.

4. Henríuuez R, Vargas NA. Historia reproductiva en puérperas de un hospital de la Región Metropolitana. Rev Méd Chile 1996; 124: 1275-80.

5. Vargas NA, Valdés M, Sabugo F, Díaz-Valdés MV, ARCE MI. Hijo no deseado. Medición de frecuen-
Un grupo particularmente afectado por el cambio fue el de las mujeres que desempeñaban alguna actividad fuera de la casa, en el que parece muy atractivo investigar las asociaciones entre ambos hechos.

No resulta ilógico suponer que detrás de estas cifras se encuentren numerosos problemas importantes para el desarrollo emocional y psicológico de los niños y para la salud mental familiar ${ }^{5-9}$. Parece razonable postular que la ruptura de lazos, secundaria al cambio, origina problemas o secuelas en quienes rompen y en sus hijos. Adicionalmente, es lógico esperar que los procesos de apego se vean comprometidos.

Con posterioridad a la ruptura, cuando ya la madre ha reconstituido una relación emocional y ha tenido otro hijo, el niño anterior se puede ver enfrentado a patrones de crianza distintos, simultáneos y, eventualmente, conflictivos. Tampoco se puede desconocer que, eventualmente, la formación de una nueva pareja y una nueva familia puede significar un regreso a patrones de convivencia mejores que los previos.

En teoría, estas situaciones pudiesen comprometer el desarrollo de las habilidades para la vida y, peor aún, generar problemas emocionales importantes en los integrantes de la familia. Todo ello merece más estudio y una reflexión profunda acerca de la forma en que se desarrollan las relaciones humanas en la sociedad chilena urbana actual.

cia y evaluación de tendencias. Rev Méd Chile 2002; 130: 638-43.

6. Centro Latinoamericano de Demografía. Comisión Económica para América Latina y el Caribe (Valeria Ramírez, Consultora). Cambios en la familia y en los roles de la mujer en América Latina y el Caribe. 1995. Santiago de Chile.

7. Salud mental desde la perspectiva pediátrica. Imágenes en salud mental. Santiago. 1999. Editada para el Congreso Psiquiatría.

8. Henríuez R, Vargas NA. Pnimera relación sexual y primer embarazo en puérperas de un hospital de la Región Metropolitana. Rev Méd Chile 1995; 123: 811-7.

9. VARGas NA. Habilidades para la vida. Pediatría al Día 2002; 18: 73-6. 